\title{
Implications of Hyperon Pairing for Cooling of Neutron Stars
}

\author{
Christoph Schaab \\ Institut für theoretische Physik, Ludwig-Maximilians Universität München, \\ Theresienstr. 37, D-80333 München, Germany; \\ schaab@gsm.sue.physik.uni-muenchen.de \\ Shmuel Balberg \\ Racah Institute of Physics, The Hebrew University, Jerusalem 91904, Israel; \\ shmblbrg@saba.fiz.huji.ac.il \\ and \\ Jürgen Schaffner-Bielich \\ Nuclear Science Division, Lawrence Berkeley National Laboratory, Berkeley, CA 94720; \\ schaffne@nta2.lbl.gov
}

\begin{abstract}
The implications of hyperon pairing for the thermal evolution of neutron stars containing hyperons are investigated. The outcome of cooling simulations are compared for neutron star models composed only of nucleons and leptons, models including hyperons, and models including pairing of hyperons. We show that lambda and neutron pairing suppresses all possible fast neutrino emission processes in not too massive neutron stars. The inclusion of lambda pairing yields better agreement with X-ray observations of pulsars. Particularly, the surface temperatures deduced from X-ray observations within the hydrogen atmosphere model are more consistent with the thermal history of neutron stars containing hyperons, if the critical temperature for the onset of lambda and nucleon pairing is not too small.
\end{abstract}

Subject headings: stars: evolution - stars: neutron

To be published in The Astrophysical Journal Letters 


\section{Introduction}

Relativistic calculations of the composition of neutron star matter lead to the conclusion that neutron stars are composed not only of nucleons and leptons but also of hyperons and, possibly, of nucleon isobars (see, e.g., Pandharipande 1971, Glendenning 1985, Schaffner \& Mishustin 1996, Balberg \& Gal 1997, Huber et al. 1997). It was proposed by Prakash et al. (1992) that the presence of hyperons might lead to rapid cooling even if the proton fraction is too small to allow for the nucleon direct Urca process and no exotic states (like meson condensates and quark gluon plasma) exist (see also Prakash 1994). This statement was confirmed by detailed cooling simulations (Haensel \& Gnedin 1994, Schaab et al. 1996). The role of hyperons in the cooling history of a neutron star is even enhanced by the generally accepted neutron pairing in the interior of the star (see, e.g., Takatsuka 1972, Amundsen \& Ostgaard 1985). Whereas the direct nucleon Urca is suppressed by neutron pairing (Lattimer et al. 1994, Page 1995) most of the hyperon processes are not. Haensel \& Gnedin (1994) and Schaab et al. (1996) found that unsuppressed rapid cooling due to hyperon induced neutrino emission processes results in surface temperatures that are too low compared with soft X-ray and extreme UV observations.

Recently Balberg \& Barnea (1998) calculated the ${ }^{1} \mathrm{~S}_{0}$ gap energy of $\Lambda$ hyperons in neutron star matter. The obtained gap energy is similar to the ${ }^{1} \mathrm{~S}_{0}$ gap energy of protons. As we will show here hyperon pairing considerably changes the cooling behavior of a neutron star containing hyperons (which we will call hyperon stars in the following) and leads to better agreement with observations.

In this Letter we study the effect of hyperon pairing on the thermal evolution of hyperon stars and compare the results with "conventional" neutron stars, which are composed of nucleons and leptons only, and with observations. We begin with the underlying physics by discussing the relevant neutrino emission processes and the pairing of hyperons (Sect. 2 and 3 ) and describing in Sect. 4 the relativistic equations of state (EOS's) used. The results of the simulations are discussed in Sect. 5. In Sect. 6 we compare our results with observations and end with some conclusions.

\section{Neutrino Emission Processes}

The early evolution of hot neutron stars is completely dominated by the cooling via neutrino emission. Only after about $10^{5}$ yrs does the photon radiation from the star's surface take over and dominate the late evolution. This late cooling might be slowed down by various heating processes (see Schaab et al. 1998b and references therein). The neutrino emission processes can be divided into slow and enhanced processes depending on whether one or two baryons participate. Due to the rather different phase spaces associated with both kind of processes the emission rates differ by several orders of magnitude.

In hyperon stars various enhanced neutrino emission processes can occur, as listed in Tab. 1. The processes are only noted in one direction. Because of $\beta$-equilibrium the inverse reaction (with $\bar{\nu}$ replaced by $\nu$ ) occurs at the same rate. The first process is the direct nucleon Urca process, the remaining processes are comprised by the term "direct hyperon Urca". The relative emissivities $\mathcal{R}_{B_{1} B_{2}}=\epsilon_{\nu}\left(B_{1} \rightarrow\right.$ $\left.B_{2}+\mathrm{e}^{-}+\bar{\nu}\right) / \epsilon_{\nu}\left(\mathrm{n} \rightarrow \mathrm{p}+\mathrm{e}^{-}+\bar{\nu}\right)$ of these processes are given with respect to the emissivity of the direct nucleon Urca process (Prakash et al. 1992). Simultaneous conservation of energy and momentum requires that the triangle inequality, $p_{B_{1}}^{\mathrm{F}}<p_{B_{2}}^{\mathrm{F}}+p_{\mathrm{e}}^{\mathrm{F}}$, and the two inequalities obtained by cyclic permutation are fulfilled for the Fermi momenta $p_{i}^{\mathrm{F}}$. If the inequalities are not fulfilled the process is extremely unlikely to occur and the corresponding emissivity vanishes. The availability of the various fast processes is thus dependent on the partial concentrations of each baryon species, which in turn is determined by the EOS.

If one of the participating baryons pairs in a superfluid state the emissivity is suppressed by an approximately exponential factor: $\mathcal{R}_{\mathrm{sf}} \sim \exp \left(-c T_{\mathrm{c}} / T\right)$ for $T \ll T_{\text {c }}$ where $T_{\text {c }}$ is the critical temperature and $c$ is a constant of the order unity. Since the Fermi momenta of the hyperons are sufficiently small they pair in a ${ }^{1} \mathrm{~S}_{0}$ state (see Sect. 3 ), as is the case for protons at slightly lower densities. Due to their high Fermi momenta, neutrons are expected to pair in an anisotropic ${ }^{3} \mathrm{P}_{2}$ state. We refer to Levenfish \& Yakovlev (1994) for fitting formulas of $\mathcal{R}_{\mathrm{sf}}$, that are valid over the whole temperature range $T<T_{\mathrm{c}}$ for both types of pairing state.

Table 1 summarizes all possible direct Urca processes with the corresponding relative emissivities. Also included are the critical density $n_{\mathrm{c}}$ and the 
Table 1: Possible direct Urca processes in hyperon stars

\begin{tabular}{cccccc} 
& & \multicolumn{2}{c}{ TM1-m1 } & \multicolumn{2}{c}{ RHF8 } \\
Process & $\mathcal{R}_{B_{1} B_{2}}$ factor & $n_{\mathrm{c}} / n_{0}$ & $M_{\mathrm{th}} / M_{\odot}$ & $n_{\mathrm{c}} / n_{0}$ & $M_{\mathrm{th}} / M_{\odot}$ \\
\hline $\mathrm{n} \rightarrow \mathrm{p}+\mathrm{e}^{-}+\bar{\nu}$ & 1 & 1.31 & 0.78 & 1.96 & 1.09 \\
$\Sigma^{-} \rightarrow \Lambda+\mathrm{e}^{-}+\bar{\nu}$ & 0.2055 & 1.89 & 1.30 & 2.99 & 1.35 \\
$\Lambda \rightarrow \mathrm{p}+\mathrm{e}^{-}+\bar{\nu}$ & 0.0394 & 1.90 & 1.31 & 3.11 & 1.38 \\
$\Sigma^{-} \rightarrow \mathrm{n}+\mathrm{e}^{-}+\bar{\nu}$ & 0.0125 & 2.37 & 1.45 & 2.44 & 1.22 \\
$\Xi^{-} \rightarrow \Lambda+\mathrm{e}^{-}+\bar{\nu}$ & 0.0175 & 2.65 & 1.52 & 6.80 & 1.65 \\
$\Sigma^{-} \rightarrow \Sigma^{0}+\mathrm{e}^{-}+\bar{\nu}$ & 0.6052 & 4.03 & 1.60 & 7.43 & $>M_{\max }$ \\
$\Xi^{-} \rightarrow \Sigma^{0}+\mathrm{e}^{-}+\bar{\nu}$ & 0.0282 & 4.03 & 1.60 & & \\
$\Xi^{-} \rightarrow \Xi^{0}+\mathrm{e}^{-}+\bar{\nu}$ & 0.2218 & 4.27 & 1.61 & & \\
$\Xi^{0} \rightarrow \Sigma^{+}+\mathrm{e}^{-}+\bar{\nu}$ & 0.0564 & 4.30 & $>M_{\max }$ & &
\end{tabular}

Note. $-\mathcal{R}_{B_{1} B_{2}}$ is the relative emissivity $\epsilon_{\nu}\left(B_{1} \rightarrow B_{2}+\mathrm{e}^{-}+\bar{\nu}\right) / \epsilon_{\nu}\left(\mathrm{n} \rightarrow \mathrm{p}+\mathrm{e}^{-}+\bar{\nu}\right), n_{\mathrm{c}}$ the critical density above which the process is allowed, and $M_{\mathrm{th}}$ the corresponding threshold mass of a non-rotating neutron star (both values depend on the underlying EOS, here: TM1-m1 and RHF8).

threshold mass of a non-rotating neutron star $M_{\mathrm{th}}$ above which the respective process is allowed for two representative EOS's (see Sect. 4). Besides the direct nucleon Urca process $\mathrm{n} \rightarrow \mathrm{p}+\mathrm{e}^{-}+\bar{\nu}$, the most important processes are $\Sigma^{-} \rightarrow \Lambda+\mathrm{e}^{-}+\bar{\nu}, \Lambda \rightarrow \mathrm{p}+\mathrm{e}^{-}+\bar{\nu}$, $\Sigma^{-} \rightarrow \mathrm{n}+\mathrm{e}^{-}+\bar{\nu}$, and $\Xi^{-} \rightarrow \Lambda+\mathrm{e}^{-}+\bar{\nu}$. Other processes are only possible at very high densities just below or above the maximum central density of a stable hyperon star. As a result, the gap energy of the $\Lambda$ particle is the most important ingredient (following those of the nucleons) for simulating the cooling history of the star, whereas the gap energies of the other hyperons need to be known only for the most massive stars. The corresponding modified hyperon Urca processes obtained by adding a bystander baryon are only important if no enhanced process is allowed since their emissivities are considerably smaller (see Maxwell 1987).

\section{Superfluidity of Hyperons}

The presence of an attractive two-body $\Lambda-\Lambda$ interaction is implied by doubly-strange hypernuclei, where two $\Lambda$ hyperons are trapped in a single nucleus (Imai 1992). The properties of the decay products of such hypernuclei indicate that the separation energy of the two $\Lambda$ hyperons, $B_{\Lambda \Lambda}$, is larger than twice the separation energy of a single $\Lambda$ from the same core nucleus, $2 B_{\Lambda}$. The bond energy derived from these experiments is $\Delta B_{\Lambda \Lambda} \equiv B_{\Lambda \Lambda}-2 B_{\Lambda} \approx 4-5 \mathrm{MeV}$, somewhat less than the corresponding bond energy of nucleons in nuclei, $B_{N N} \approx 6-7 \mathrm{MeV}$. Hence, $\Lambda$ hyperon pairing similar to that of nucleons is indeed expected in high density matter.

In our simulations presented below we use the $\Lambda$ hyperon ${ }^{1} \mathrm{~S}_{0}$ gaps as estimated recently by Balberg \& Barnea (1998). Their $\Lambda \Lambda$ potential is based on the $G$-Matrix parameterization (Lanskoy \& Yamamoto 1997), which accurately reproduces the bond energy of various doubly strange hypernuclei. In this model, the pairing energy is calculated as a function of the $\Lambda$ Fermi momenta and the background baryon density, while neglecting relativistic and polarization effects.

In general, $\Lambda$ pairing was found to extend from the threshold density for $\Lambda$ formation to the density where the $\Lambda$ Fermi momenta exceeds $1.3 \mathrm{fm}^{-1}$ (typically corresponding to a $\Lambda$ fraction of $0.15-0.2$ ). The corresponding gap energies were found to be several tenths of a $\mathrm{MeV}$, where the exact value depends on both the $\Lambda$ fraction and the background matter density. These values are actually similar to those found for proton ${ }^{1} \mathrm{~S}_{0}$ pairing with a neutron matter background (Elgarøy et al. 1996), again indicating the general similarity between hyperon-related and nucleon related interactions expected at supernuclear densities.

The pairing gaps are also strongly dependent on the effective mass of the particles on the Fermi surface, since the pairing interaction competes with the particles' kinetic energy. Relativistic calculations typically predict that at supernuclear densities the the baryon effective masses are considerably lower than the bare masses, but the exact value on the Fermi 


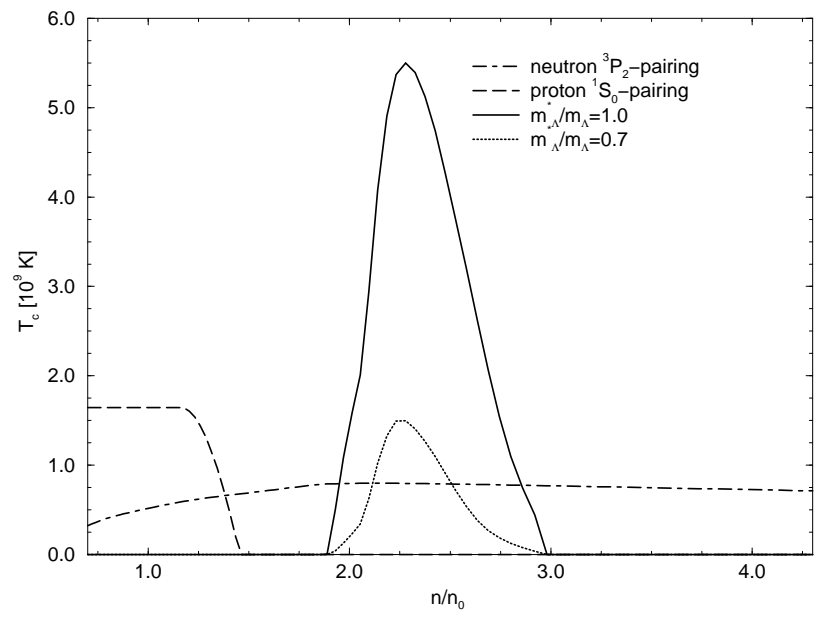

Fig. 1.- Critical temperatures for the TM1-m1 EOS.

surface is highly model dependent. We thus treat the value of the effective mass as a parameter, which allows to examine the sensitivity of our results to the uncertainty in the $\Lambda$ gap energies.

Figure 1 shows the critical temperature for $\Lambda$ ${ }^{1} \mathrm{~S}_{0}$ pairing expected for the equilibrium compositions found with the TM1-m1-model EOS (Schaffner $\&$ Mishustin 1996). The critical temperature $T_{\mathrm{c}}$ is related to the gap energy $\Delta(0)$ at zero temperature by $T_{\mathrm{c}}=a \Delta(0) / k_{\mathrm{B}}$, where the proportional factor $a$ depends on the pairing type (see Levenfish \& Yakovlev 1994). Note the strong dependence of the critical temperature on the $\Lambda$ effective mass, $m_{\Lambda}^{*}$, (unlike the density range in which ${ }^{1} \mathrm{~S}_{0}$ paring exists, which is practically independent of $m_{\Lambda}^{*}$ ). We show the critical temperatures when the $\Lambda$ mass on the Fermi surface is taken as equal to the bare one, $m_{\Lambda}^{*} / m_{\Lambda}=1$, and also when this mass is reduced by a factor of $m_{\Lambda}^{*} / m_{\Lambda}=0.7$. The latter case is a reasonable representation for the value of the bulk effective mass as found in the selfconsistent calculation of the TM1-m1 model. We stress again that the mass on the Fermi surface may differ considerably from the bulk effective mass, and the two cases will be used here mainly to examine the dependence of the thermal history on the $\Lambda$ critical temperature. Also shown are the critical temperatures for proton ${ }^{1} \mathrm{~S}_{0}$ and neutron ${ }^{3} \mathrm{P}_{2}$ pairing, as calculated by Wambach et al. (1991) and Amundsen \& Østgaard (1985), respectively. Note that neutron ${ }^{3} \mathrm{P}_{2}$ pairing exists over the entire supernuclear density range in this specific model.

\section{Equations of State}

The EOS is quite unknown at high density. For the present study, we choose two recent relativistic parameterizations, which have been proven to be able to describe properties of nuclei and/or nucleon scattering data.

The first set (TM1-m1) is a relativistic mean-field calculation (model 1 of Schaffner \& Mishustin 1996). The nucleon parameters are adjusted to properties of nuclei (set TM1, see Sugahara \& Toki 1994). The vector potential is nonlinear in density to get a soft behavior at high density in accordance with DiracBrückner calculations. The hyperon coupling constant are fixed by $\mathrm{SU}(6)$ symmetry and hypernuclear data.

The other set (RHF8 from Huber et al. 1997) uses Relativistic-Brückner-Hartree-Fock results up to 2-3 times normal nuclear density. Hyperons are implemented within the Relativistic-Hartree-Fock approach. The EOS calculated within RelativisticHartree-Fock are usually much softer than those derived from relativistic mean-field models due to the additional degrees of freedom. Again, SU(6) symmetry as well as hypernuclear data are used to fix the hyperon coupling constants.

The hyperons appear at a slightly higher density for RHF8 than in TM1-m1 except for the $\Sigma^{-}$which appears around the same density of $2 \rho_{0}$. The maximum fraction of $\Sigma^{-}$is higher in RHF8 than in TM1$\mathrm{m} 1$, while the $\Lambda$ fraction is about a factor of two smaller. This has an impact on the onset of the various hyperon cooling processes as seen in Tab. 1. The critical density for hyperon induced cooling processes is usually lower for TM1-m1 than for RHF8, except for the process $\Sigma^{-} \rightarrow \mathrm{n}+\mathrm{e}^{-}+\bar{\nu}$, which is however suppressed by neutron pairing.

\section{Results}

The general relativistic equations of stellar structure and thermal evolution (see Thorne 1977) were numerically solved via an implicit finite difference scheme by a Newton-Raphson algorithm (see Schaab et al. 1996 for more details) ${ }^{1}$. The surface temperatures deduced from the observed thermal X-ray spec-

\footnotetext{
${ }^{1}$ Tables with detailed references to the used ingredients, the used observational data, and the obtained cooling tracks can be found on the Web: http://www.physik.uni-muenchen.de/sektion/suessmann/astro/cool/schaab.0897.
} 


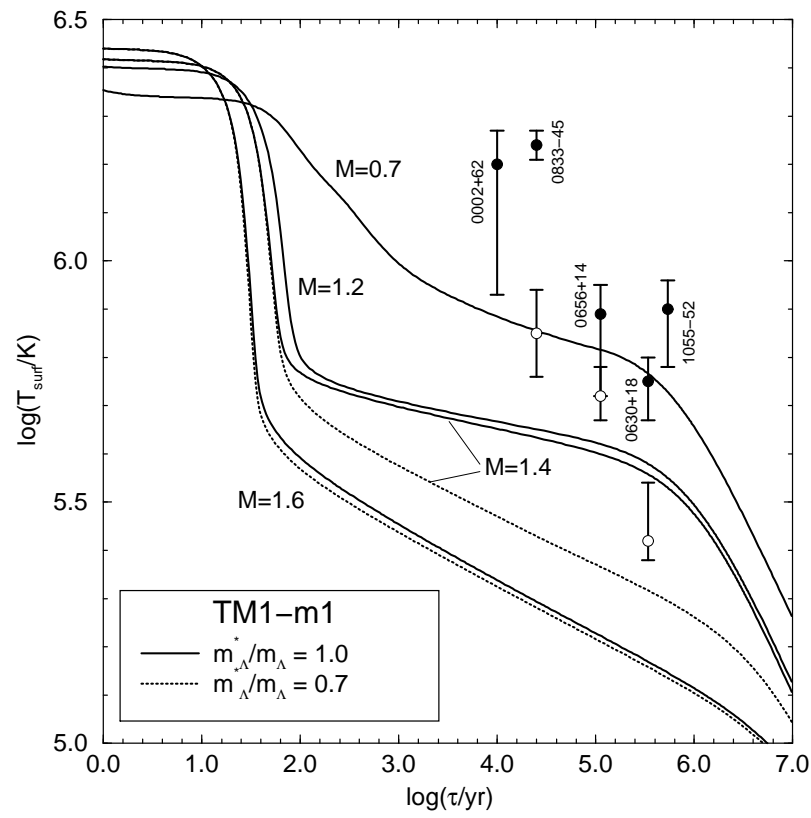

Fig. 2.- Cooling of neutron and hyperon stars constructed for the TM1-m1 EOS. The observational data obtained within the magnetic hydrogen atmosphere model are marked by hollow circles, whereas the one obtained within the pure iron atmosphere model are marked by solid ones.

tra depend on the used atmosphere model. We plot the surface temperatures along with their $2 \sigma$ error bars for the two extreme cases of a pure iron atmosphere (solid circles in Figs. 2 and 3) and of a magnetic hydrogen atmosphere (hollow circles, see Schaab et al. 1998a for more details). The deduced surface temperatures can only be understood as upper limits to the evolution temperature, since magnetospheric emission may irradiate the star's surface. On the other hand, the magnetic hydrogen atmosphere model is likely to underestimate the actual surface temperature (Potekhin et al. 1997). The two extreme values of the respective star's surface temperature should therefore give a good estimate of the maximum range of the true evolution temperature.

Fig. 2 shows the cooling behavior of hyperon stars constructed for the TM1-m1 EOS. The surface temperature as measured by a distant observer is plotted against the star's age for different star masses (a larger mass corresponds to a larger central density, thus crossing the thresholds of more cooling processes; see Lattimer et al. 1991).
- $M=0.7 M_{\odot}$ : This model is an example for a slow cooling neutron star. The interior does not contain hyperons, nor is the proton fraction sufficiently high to allow for the direct nucleon Urca. Note that the relatively low surface temperature is caused by the inclusion of the superfluid pair breaking and formation process (Voskresenskii \& Senatorov 1987, Schaab et al. 1997), which is not included in former works (e.g. Umeda et al. 1994, Page 1995, Schaab et al. 1996).

- $M=1.2 M_{\odot}$ : The interior consists still only of nucleons, but the nucleon direct Urca is now possible. This yields a faster cooling which is slowed down by neutron pairing below the critical temperature $T_{\mathrm{c}} \approx 8 \times 10^{8} \mathrm{~K}$.

- $M=1.4 M_{\odot}:$ At $n \approx 1.8-1.9 n_{0} \Sigma^{-}$and $\Lambda$ hyperons begin to populate the central region of the star. Besides the direct nucleon Urca the processes $\Sigma^{-} \rightarrow \Lambda+\mathrm{e}^{-}+\bar{\nu}$ and $\Lambda \rightarrow \mathrm{p}+\mathrm{e}^{-}+\bar{\nu}$ are also possible. The two direct hyperon processes are suppressed by $\Lambda$ pairing below the critical temperature $T_{\mathrm{c}}$. At the outer boundary of the hyperon core $T_{\mathrm{c}}$ is rather small $\left(\approx 10^{8} \mathrm{~K}\right.$ for $m_{\Lambda}^{*} / m_{\Lambda}=1$ and $\approx 10^{7} \mathrm{~K}$ for $\left.m_{\Lambda}^{*} / m_{\Lambda}=0.7\right)$. The hyperon processes are therefore significantly suppressed only in the innermost part of the star. The surface temperature of a middle aged, enhanced cooling neutron star $\left(10^{2} \mathrm{yrs} \lesssim \tau \lesssim 10^{5} \mathrm{yrs}\right)$ is directly related to the critical temperature of its superfluids (Lattimer et al. 1994, Page 1995, Schaab et al. 1996) and depends therefore on the considered gap energy model. For the $\Lambda$ gap model with $m_{\Lambda}^{*} / m_{\Lambda}=1$, the critical temperature of $\Lambda$ pairing is larger than for neutron ${ }^{3} \mathrm{P}_{2}$ pairing. In this specific model, the cooling behavior is therefore determined by the critical temperature for the latter.

- $M=1.6 M_{\odot}:$ At densities $n \gtrsim 3 n_{0}$ the scalar $\Lambda \Lambda$ interaction becomes repulsive in our model TM1-m1, and the $\Lambda$ 's no longer pair in a ${ }^{1} \mathrm{~S}_{0}$ state. The direct hyperon Urca processes are therefore unsuppressed and the interior of the star cools very rapidly. This result relies however on the specific assumptions that we neglected pairing of other hyperons $\left(\Sigma^{0, \pm}, \Xi^{0,-}\right)$ and pairing of $\Lambda$ in a ${ }^{3} \mathrm{P}_{2}$ state. If such further 


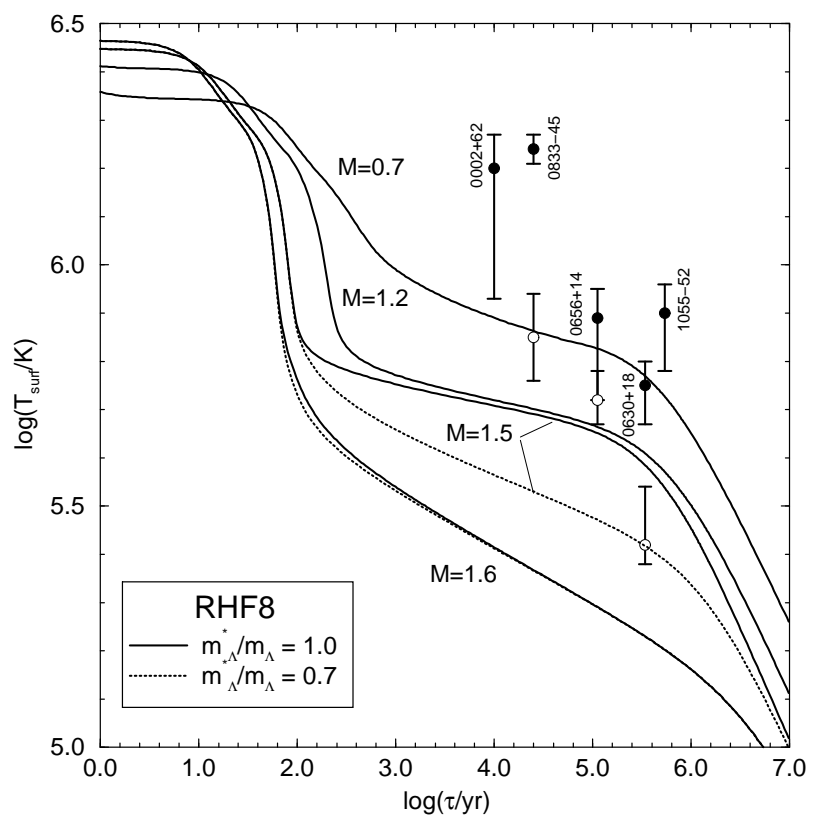

Fig. 3.- Same as Fig. 2 but for the RHF8 EOS.

pairing is allowed the cooling behavior should be similar to that of a $M=1.4 M_{\odot}$ star.

Fig. 3 shows the cooling behavior for the RHF8 EOS. As for TM1-m1 we have four scenarios corresponding to the four star masses. The cooling behavior of these scenarios are quite similar for both cases of EOS's, although some aspects are somewhat model dependent (TM1-m1 stars generally cool more rapidly). We emphasize the effect of $\Lambda$ pairing on the thermal evolution for both EOS's, which is explicit in the difference between the $M=1.4 M_{\odot}\left(M=1.5 M_{\odot}\right.$ for RHF8) thermal sequences and the $M=1.6 M_{\odot}$ sequences, as the latter essentially assume no hyperon pairing. This effect is enhanced, of course, for larger $\Lambda$ pairing energies.

\section{Conclusions and Discussion}

In this Letter we have studied the effect of hyperon pairing on the thermal evolution of hyperon stars. Our general finding is that $\Lambda$-hyperon pairing along with nucleon pairing is sufficient to suppress fast hyperon-induced cooling processes in all but the most massive neutron stars, in a similar fashion as the suppression of fast nucleon processes by nucleon pairing. Pairing of other hyperons becomes important only for cooling of the most massive stars with
$M>1.6 M_{\odot}$.

As a result of this suppression, the surface temperatures obtained in cooling simulations of hyperon stars are considerably higher than those found for the same stars when the $\Lambda$ hyperons are taken to be in a normal state. In particular, this allows better compatibility with surface temperatures deduced within the magnetic hydrogen atmosphere model from observed thermal emission from pulsars, with which cooling of hyperon stars through unsuppressed rapid cooling has been previously found to be inconsistent (Haensel \& Gnedin 1994, Schaab et al. 1996).

Our quantitative results demonstrate a significant sensitivity of the surface temperatures to the modeling of the hyperon pairing. We also call attention to the dependence of the surface temperature on the details of the high density EOS through the equilibrium concentrations of the baryon species. In this sense, comparison of the outcome of theoretical cooling models with soft X-ray and extreme UV observations provides a powerful tool for investigating the interiors of pulsars. Future measurement of pulsar thermal emission may indeed offer valuable indication regarding the physics and composition of veryhigh density matter, although much improvement is still necessary in both theory and observation.

Our main conclusion is that that the existence of hyperons in neutron stars cannot be excluded by comparing their predicted thermal history with actual observations from pulsars, provided the hyperons are allowed to form a superfluid state - similar to that of the nucleons. Though we have neglected several effects, such as medium effects on the modified Urca process, possible meson condensation and accreted envelopes, we believe this main conclusion to be quite robust. The study of these effects, as well as pairing of other hyperon species and other hyperon coupling models will be addressed in future work.

Ch. S. gratefully acknowledges the Bavarian State for financial support. J. S.B. is supported by the Alexander von Humboldt-Stiftung and by the U.S. Department of Energy under Contract No. DE-AC0376SF00098.

\section{REFERENCES}

Amundsen, L. \& Østgaard, E. 1985, Nucl. Phys. A 442, 163

Balberg, S. \& Barnea, N. 1998, Phys. Rev. C, 57, 409 
Balberg, S. \& Gal, A. 1997, Nucl. Phys. A, 625, 435

Elgarøy, Ø., Engvik, L., Hjorth-Jensen, M. \& Osnes, E. 1996, Phys. Rev. Lett., 77, 1428

Glendenning, N. K. 1985, Astrophys. J., 293, 470

Haensel, P. \& Gnedin, O. Y. 1994, Astron. Astrophys., 290, 458

Huber, H., Weber, F., Weigel, M. K., \& Schaab, C. 1997, preprint nucl-th/9711025, to be published in Int. J. of Mod. Phys. E

Imai, K. 1992, Nucl. Phys. A, 547, 653

Lanskoy, D. E. \& Yamamoto, Y. 1997, Phys. Rev. C, $\mathbf{5 5}, 2330$

Lattimer, J. M., Pethick, C. J., Prakash, M. \& Haensel, P. 1991, Phys. Rev. Lett. 66, 2701

Lattimer, J. M., Van Riper, K. A., Prakash, M. \& Prakash, M. 1994, Astrophys. J. 425, 802

Levenfish, K. \& Yakovlev, D. 1994, Pisma Astron. Zh., 20, 54

Maxwell, O. V. 1987, Astrophys. J., 316, 691

Page, D. 1995, Revi. Mex. Fís., 41, Supl. 1, 178

Pandharipande, V. 1997, Nucl. Phys. A, 178, 123

Potekhin, A. Y., Chabrier, G. \& Yakovlev, D. G. 1997, A \& A 323, 413

Prakash, M., Prakash, M., Lattimer, J., \& Pethick, C. 1992, Astrophys. J., 390, L77

Prakash, M. 1994, Phys. Rep. 242, 297

Schaab, C., Weber, F., Weigel, M. K., \& Glendenning, N. K. 1996, Nucl. Phys. A, 605, 531

Schaab, C., Voskresenskii, D., Sedrakian, A.D., Weber, F. \& Weigel, M.K. 1997, A \& A 321, 591

Schaab, C., Weber, F., \& Weigel, M.K. 1998a, A \& A $\mathbf{3 3 5}, 596$

Schaab, C., Sedrakian, A. D., Weber, F., \& Weigel, M. K. 1998b, in preparation

Schaffner, J. \& Mishustin, I. N. 1996, Phys. Rev. C, $\mathbf{5 3}, 1416$
Sugahara, Y. \& Toki, H. 1994, Nucl. Phys. A, 579, 557

Takatsuka, T. 1972, Prog. Theor. Phys., 48, 1517

Thorne, K. 1977, Astrophys. J., 212, 825

Umeda, H., Tsuruta, S. \& Nomoto, K. 1994, Astrophys. J., 433, 256

Voskresenskii, D.N. \& Senatorov, A.V. 1987, Sov.J.Nucl.Phys. 45, 411

Wambach, J., Ainsworth, T. L. \& Pines D. 1991, in Ventura, J., Pines, D. (eds.), Neutron Stars: Theory and Observation, Kluwer Academic Publishers, Dordrecht (Netherlands), pp. 37

This 2-column preprint was prepared with the AAS LATEX macros $\mathrm{v} 4.0$. 